\title{
A influência da espessura da resina composta de esmalte e dentina na cor de restaurações anteriores
}

\author{
The influence of enzyme and dentine resin composit thickness in the color of previous
} restorations

\author{
Thamiris Sant'Ana Martins ${ }^{1}$ \\ Antônia Patrícia Oliveira Barros ${ }^{2}$ \\ Cristiane de Melo Alencar ${ }^{3}$ \\ Mariangela Ivette Guanipa Ortiz ${ }^{3}$ \\ Jesuína Lamartine Nogueira Araújo ${ }^{4}$ \\ Eliane Bemerguy Alves ${ }^{4}$
}

\section{RESUMO}

O estudo consistiu em uma análise laboratorial para avaliar o grau de compatibilidade de cor da resina composta da marca Opallis FGM em relação à escala de cores VITA Classical. Foram confeccionados corpos de prova de resina composta para esmalte nas cores EA1, EA2, EA3, A3,5, com espessuras de 0,$2 ; 0,4 ; 0,6 ; 0,8$ e $1 \mathrm{~mm}$ e resina para dentina DA2 e DA3,5 nas espessuras de 1 e $2 \mathrm{~mm}$. Os dados a respeito da compatibilidade de cor foram mensurados através do espectrofotômetro Vita Easyshade Advance. Foram analisados os valores de $\Delta \mathrm{E}$ adotando-se como referência o valor de $\Delta \mathrm{E} \leq 3,3$ para semelhança com o padrão da escala VITA. Os resultados foram analisados através do Programa Bioestat - versão 5.3 sendo submetidos à ANOVA e Tukey. Foi observada a tendência de diminuição dos valores de $\Delta \mathrm{E}$ à medida que se aumentava a espessura de resina. Concluiu-se ser imprescindível a associação de resinas de esmalte e dentina para obtenção de compatibilidade de cor com o padrão da escala VITA Classical.

Palavras-chave: Restauração Dentária. Espectrofotometria. Estética Dentária. Cor.

\section{ABSTRACT}

The study consisted of a laboratory analysis evaluating the degree of color compatibility of the composite resin of the brand Opallis FGM in relation to the Vita Classical color scale. Composite resin specimens were prepared for enamel and dentin in the colors EA1, EA2, EA3, A3,5, DA2 and DA3,5. Data on resin color compatibility with Vita color scale were counted using the Vita Easyshade Advance spectrophotometer. The values collected were $\Delta \mathrm{E} ; \mathrm{L} ; \mathrm{C} ; \mathrm{H}$ and ${ }^{*}$, with only the values of $\Delta \mathrm{E}$ considered for analysis. The value of $\Delta \mathrm{E} \leq 3.3$ was adopted as reference for similarity to the VITA scale standard. The results were analyzed through the Bioestat Programversion 5.3. The decrease tendency of $\Delta \mathrm{E}$ was observed as the resin thickness was increased, but acceptable numbers were only achieved in the combinations. It was concluded that it is essential to associate enamel and dentin resins to obtain acceptable values.

Keyword: Dental Restauration. Spectrophotometry. Esthetics dental. Color.

\footnotetext{
${ }^{1}$ Cirurgião-dentista.

${ }^{2}$ Estudante de Graduação, Faculdade de Odontologia, Universidade Federal do Pará

3 Pós-graduanda, Faculdade de Odontologia, Universidade Federal do Pará

${ }^{4}$ Professor(a) Associada, Faculdade de Odontologia, Universidade Federal do Pará
} 


\section{INTRODUÇÃ̃o}

Os tratamentos restauradores estéticos são procedimentos que fazem parte de grande número de atendimentos nos consultórios odontológicos. Para que estas restaurações sejam esteticamente satisfatórias, precisam apresentar forma, textura e cor compatíveis com o aspecto natural de um dente hígido ${ }^{1}$. Restaurações que não se enquadram nestes quesitos, podem tanto deixar o cirurgião dentista frustrado com o resultado do trabalho, como não atingir a expectativa dos pacientes.

Para melhor entender o processo restaurador, é preciso conhecer conceitos relacionados à cor. Esta pode ser definida como uma sensação que a ação de luz provoca sobre o órgão da visão ${ }^{2}$, portanto, sua existência está atrelada à existência de luz, não sendo algo que exista materialmente ${ }^{3,4}$. A cor é definida como uma decorrência da interação dos raios luminosos com a superfície de um objeto ${ }^{5}$. Desta forma, os objetos não possuem cor, e sim, apresentam capacidade de absorver, refratar ou refletir raios luminosos que incidam sobre eles.

A cor é entendida como uma estrutura tridimensional composta em: matiz, croma e valor ${ }^{6}$. Segundo o sistema de ordenamento de cor de Munsell, a matiz é a qualidade que nos permite distinguir uma família de cor da outra, como vermelho do amarelo, do azul ou do roxo. Croma é a intensidade da cor nos permitindo distinguir uma cor forte de uma cor fraca, ou seja, é definido como a saturação de um determinado matiz. Valor é o que diferencia uma cor clara de uma escura, sendo conceituada como a quantidade de preto e branco em um objeto "[...] este fenômeno define a vitalidade de um corpo, provocando sensações de profundidade ou aproximação" 7 .

Nas resinas, estes conceitos refletem na classificação de suas cores. A mais conhecida e utilizada é a escala de cores VITA Classical (Vita Zahnfabrik, Berlin, DE), onde o matiz determina os grupos de resina A (marrom), B (amarelo com pouco marrom), C (cinza com pequena quantidade de marrom) e D (rosa avermelhado com um pouco de marrom). O croma é representado pela numeração gradual após a letra, variando de 1 a 4 na maioria das marcas comerciais. $\mathrm{O}$ valor está relacionado à quantidade de opacidade e translucidez nas resinas compostas, diferenciando o uso de resinas para esmalte e dentina ${ }^{8}$.

As resinas são compostas basicamente de matriz orgânica (monômeros, inibidores, modificadores de cor e sistema iniciador/ativador), inorgânica (quartzo, sílica) e radiopacificadores (bário e estrôncio). A matriz orgânica pode ser composta de Bis-GMA, UDMA, TEGDMA, EGDMA. Em temperatura ambiente, são materiais altamente viscosos gerando dificuldades de incorporação de carga inorgânica à matriz resinosa, por isso é necessária a presença de agentes de união. A matriz inorgânica tem a função de aumentar as propriedades mecânicas, diminuindo a contração de polimerização e o coeficiente de expansão térmica, já que são dimensionalmente estáveis ${ }^{9}$.

Devido à dificuldade de adesão das partículas à matriz orgânica, é adicionado um silano como agente de união. Ele pode unir-se quimicamente tanto às partículas, como à matriz orgânica. Isso faz com que ocorra uma interface adesiva satisfatória e confiável. Essa interface atua dissipando as tensões as quais a resina composta é submetida, atuando como uma unidade, evitando fraturas. Os agentes iniciadores são compostos de canforoquinona que quando ativada pela luz visível, com comprimento de onda de cerca de 470 $\mathrm{nm}$, libera radicais livres, os quais levarão ao início da reação de polimerização ${ }^{10}$.

Há uma grande variedade de resinas compostas no mercado odontológico e devido à crescente demanda de uso nos procedimentos clínicos, vários estudos foram realizados para que a tecnologia fosse aprimorada visando um material adequado, tanto mecânico como esteticamente. Utilizando a classificação de acordo com tamanho médio das partículas, podemos encontrar as resinas macroparticuladas, microparticuladas, híbridas, microhíbridas, nanoparticuladas e nanohíbridas ${ }^{9}$.

Com o aumento do apelo estético, além das propriedades mecânicas, propriedades físicas e ópticas tiveram que ser também colocadas em destaque na produção de resinas. Tais propriedades têm sido bastante estudadas pelos fabricantes de resinas, já que tentar imitar as propriedades ópticas de um dente natural não é algo simples. O esmalte apresenta características mais translúcidas e não é fácil de ser reproduzido em uma restauração anterior, pois suas características dependem diretamente do nível de desgaste a que os dentes naturais 
foram submetidos. Por outro lado, a dentina apresenta características de maior opacidade e tem grande importância no momento de estratificar uma restauração. Portanto, resinas translúcidas serviriam como um esmalte artificial e as resinas opacas, como uma dentina artificial. Partindo-se deste princípio, deveríamos determinar a área de aplicação de cada tipo de resina composta seguindo características de comportamento mecânico e óptico, bem como a espessura destas camadas, a fim de potencializar o resultado estético ${ }^{7,11}$.

Atualmente, encontram-se no mercado odontológico resinas com características ópticas que tentam simular as propriedades naturais do esmalte e da dentina. O padrão proposto para sua utilização, preconiza a inserção de resina de dentina nas regiões do preparo cavitário em substituição ao conteúdo de dentina perdida, e o mesmo para as resinas de esmalte que entram como as últimas camadas, fazendo às vezes de esmalte dental.

Com o objetivo de observar o desempenho estético destas resinas, no que se refere à compatibilidade de cor com a estabelecida pela escala de cor Vita Classical (VITA Zahnfabrik ${ }^{\circledR}$ ), realizou-se um estudo laboratorial por meio da espectrofotometria

\section{Materiais e Métodos}

O estudo consistiu em uma análise laboratorial que avaliou o grau de compatibilidade de cor da resina composta da marca Opallis (FGM, Joinville, SC, BR) em relação à escala de cores Vita Classical (VITA Zahnfabrik $($ )). Foram confeccionados corpos de prova de resina composta de esmalte e dentina. Cada um apresentou aproximadamente $1 \mathrm{~cm}$ de diâmetro, nas cores para dentina DA2 e DA3,5 e esmalte EA1, EA2, EA3 e EA3,5. Para cada cor foram feitas 3 amostras. As amostras de resina de dentina foram confeccionadas em espessuras de $1 \mathrm{~mm}$ e $2 \mathrm{~mm}$ e as de esmalte em $0,2 \mathrm{~mm}$; $0,4 \mathrm{~mm} ; 0,6 \mathrm{~mm} ; 0,8 \mathrm{~mm}$ e $1 \mathrm{~mm}$, totalizando 72 corpos de prova.

Os corpos de prova foram confeccionados utilizandose uma matriz de Polivinil Siloxano (Express XT, 3M ESP, St. Paul, MN, USA), colocando-se incrementos únicos de resina entre duas tiras de poliéster prensadas por duas placas de vidro. A obtenção das diferentes espessuras foi feita pela interposição de folhas de papel com espessura de 0,01 mm, colocadas em ambas as extremidades da placa de vidro de maneira simétrica. Depois da remoção de excessos, que por ventura ultrapassassem os limites das tiras de poliéster, os incrementos foram fotopolimerizados (LEDLUX II Ortus, Campo Mourão, PR, BR) posicionando a ponteira sobre as amostras em três fotoativações de $500 \mathrm{~mW} / \mathrm{cm}^{2}$, no tempo de 20 segundos cada, de modo que abrangesse toda a extensão do corpo de prova. Para garantir a padronização das espessuras, os corpos de provas foram aferidos por um paquímetro digital (Starret, série 727, Itú, SP, BR). Os corpos de prova foram armazenados em recipientes herméticos, em total ausência de luz, por um período de 24 horas, até que fossem feitas as leituras da cor.

Os dados relativos à compatibilidade de cor da resina em relação à escala de cor Vita foram observados através do espectrofotômetro Vita Easyshade Advance (VITA Zahnfabrik®) na função "verificar restauração", após a devida calibração do aparelho.

Para a leitura da cor, as amostras foram posicionadas sobre uma superfície totalmente branca, minimizando possíveis interferências de cor. Foram realizadas leituras de cada cor de resina de esmalte e de dentina individualmente em todas as espessuras e também das associações destes dois tipos de resina, sempre superpondo o esmalte sobre a dentina e fazendo a leitura do conjunto perfazendo 80 diferentes associações. Em cada corpo de prova individualmente e em associações foram feitas três aferições para posteriormente calcular a média aritmética dos valores de $\Delta \mathrm{E}$. Os registros foram coletados e fixados em planilhas do programa Microsoft Office Excel 2016 para posterior análise estatística.

Adotou-se como referência o valor de $\Delta \mathrm{E} \leq 3,3$ para semelhança com o padrão da escala VITA. Valores de $\Delta \mathrm{E}$ acima de 3,3 são considerados como diferenças perceptíveis ao olho humano ${ }^{12,13}$. Foram realizados os testes estatísticos de Shapiro Wilk, ANOVA e Tukey. 


\section{Resultados}

\subsection{Avaliação individual de diferentes cores e espessuras}

Os resultados foram analisados através do Programa Bioestat ${ }^{\circledR}$, versão 5.3 (Instituto Mamirauá, AM, BR). Para todas as comparações foi considerado um nível de significância de 5\% $(\alpha=0,05)$.

O teste de Shapiro-Wilk demonstrou distribuição normal $(p \geq 0,05)$ dos dados individuais de $\Delta E$ para todos os compósitos avaliados.

Mediante análise descritiva dos dados individuais foi observada a diminuição progressiva da média aritmética $(\bar{\chi})$ dos valores de $\Delta \mathrm{E}$ conforme era aumentada a espessura dos compósitos tanto de dentina (DA2 e DA3,5) quanto de esmalte (EA1, EA2, EA3 e EA3,5) (Tabelas 1 e 2).

Tabela 1. Média $(\bar{\chi})$ e Desvio padrão (DP) dos valores de $\Delta \mathrm{E}$ em dentina

\begin{tabular}{|c|c|c|}
\hline $\begin{array}{c}\text { Espessura } \\
\text { Cor }\end{array}$ & $1 \mathrm{~mm}$ & $2 \mathrm{~mm}$ \\
\hline DA2 & $12,7( \pm 0,0)$ & $8,5( \pm 1,0)$ \\
\hline DA3,5 & $15,6( \pm 0,2)$ & $7,6( \pm 0,4)$ \\
\hline
\end{tabular}

Fonte: UFPA, 2017.

Tabela 2. Média $(\bar{\chi})$ e Desvio padrão (DP) dos valores de $\Delta \mathrm{E}$ para os compósitos de esmalte em diferentes cores e espessuras.

\begin{tabular}{cccccc}
\cline { 1 - 4 } Espessura & $0,2 \mathrm{~mm}$ & $0,4 \mathrm{~mm}$ & $0,6 \mathrm{~mm}$ & $0,8 \mathrm{~mm}$ & $1 \mathrm{~mm}$ \\
\cline { 1 - 1 } Cor & & & & & \\
\cline { 1 - 5 } EA1 & 12,4 & 11,3 & 7,3 & 5,9 & 5,5 \\
& $( \pm 0,8)$ & $( \pm 1,2)$ & $( \pm 0,9)$ & $( \pm 0,4)$ & $( \pm 0,4)$ \\
EA2 & 15,9 & 13,2 & 11,1 & 7,0 & 6,5 \\
& $( \pm 1,1)$ & $( \pm 0,4)$ & $( \pm 0,8)$ & $( \pm 0,2)$ & $( \pm 0,3)$ \\
EA3 & 15,8 & 13,6 & 10,3 & 7,7 & 6,5 \\
& $( \pm 0,6)$ & $( \pm 0,2)$ & $( \pm 0,9)$ & $( \pm 0,2)$ & $( \pm 0,1)$ \\
EA3,5 & 17,0 & 15,9 & 14,9 & 13,7 & 11,6 \\
& $( \pm 0,7)$ & $( \pm 0,3)$ & $( \pm 0,5)$ & $( \pm 0,6)$ & $( \pm 0,2)$ \\
\hline
\end{tabular}

Fonte: UFPA, 2017.

\subsection{Avaliação da interação de diferentes cores e espessuras}

Para avaliação da interação dos compósitos, em diferentes cores e espessuras, foi realizada análise descritiva dos dados em comparação aos parâmetros clinicamente aceitáveis, ou seja, compatíveis com o determinado na escala VITA, representado por $\Delta \mathrm{E} \leq 3,3$.
3.2.1- Interações entre resinas de dentina DA2 com as diferentes saturações de esmalte

Para avaliação da diferença entre as médias dos diferentes grupos determinados pelas interações da resina de dentina com a de esmalte (EA1 x DA2, EA2 x DA2, EA3 x DA2 e EA3,5 x DA2) foi utilizada a análise de variância (ANOVA) one way, seguida do pós-teste de Tukey. Devido à ausência de homocedasticidade (variâncias semelhantes), foi necessário excluir da análise a comparação DA2 x EA3,5.

O F obtido foi altamente significativo $(\mathrm{p}=0,0002)$, apontando nove comparações com diferença estatisticamente significante $[(1 \times 2) ;(1 \times 4) ;(1 \times 6) ;(2 \times 3)$; $(2 \times 5),(3 \times 4) ;(3 \times 6)$ e $(4 \times 5)$ e $(5 \times 6)]$ (Tabela 3$)$. As demais comparações entre as combinações de compósitos não apresentaram diferença estatística. Com relação à comparação das diferentes espessuras de resina de esmalte, o teste acusou diferença estatística $(p<0,005)$ entre as amostras [(1x3); $(1 \times 4) ;(1 \times 5)]$. Os resultados ainda demonstraram maior compatibilidade com o padrão VITA quando se utilizou a camada mais espessa (2 mm) do compósito DA2, combinada a diferentes espessuras de resina para esmalte, quando comparado à espessura de $1 \mathrm{~mm}$ de resina de dentina, cujos dados estão expostos na Tabela 3.

Tabela 3 Média $(\bar{\chi})$ e Desvio padrão (DP) dos valores de $\Delta \mathrm{E}$ para a combinação dos compósitos em diferentes e espessuras.

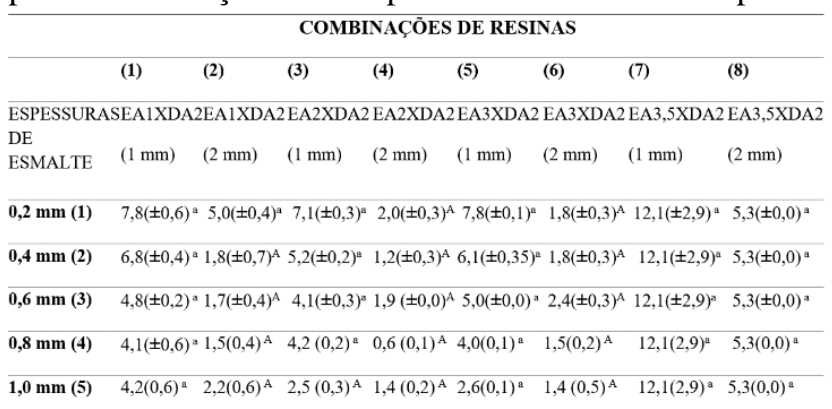

Fonte: UFPA, 2017.

Letra maiúscula (A) para valores compatíveis com a Escala VITA $(\Delta E \leq 3,3)$; Letra minúscula (a) para valores não compativeis com a Escala VITA $(\Delta E>3,3)$. 


\subsubsection{Interações entre resina de dentina DA3,5 com as diferentes saturações de esmalte.}

Para avaliação da diferença entre as médias dos diferentes grupos de interações da resina de dentina com as de esmalte (EA1 x DA3,5, EA2 x DA3,5, EA3 $\mathrm{x}$ DA3,5 e EA3,5 x DA3,5) foi utilizada a análise de variância (ANOVA) two way, seguida do Teste de Tukey. $O$ valor de $F$ obtido foi significativo ( $\mathrm{p}=0,0021)$, apontando seis comparações estatisticamente

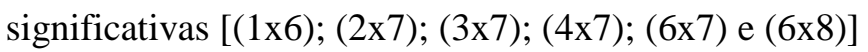
(Tabela 4). As demais comparações entre as combinações de compósitos não apresentaram diferença estatística. Com relação à comparação das diferentes espessuras de resina de esmalte, o teste acusou diferença estatística $(\mathrm{p}<0,005)$ entre as amostras [(1x3); (1x4); $(1 \times 5)]$.

$\mathrm{Na}$ avaliação da interação dos compósitos de dentina DA3,5 foi verificado que a camada mais espessa deste compósito $(2 \mathrm{~mm})$ combinada a diferentes espessuras de resina para esmalte, apresentaram os resultados mais próximos ao padrão VITA do que na espessura de $1 \mathrm{~mm}$. Exceção foi observada na combinação DA3,5 X EA3,5 onde não houve equivalência de cor em praticamente todas as associações (Tabela 4).

Tabela 4. Média $(\bar{\chi})$ e Desvio padrão (DP) dos valores de $\Delta \mathrm{E}$ para a combinação dos compósitos em diferentes cores e espessuras.

\begin{tabular}{|c|c|c|c|c|c|c|c|c|}
\hline \multicolumn{9}{|c|}{ COMBINAÇÕES DE RESINAS } \\
\hline & (1) & (2) & (3) & (4) & (5) & (6) & (7) & (8) \\
\hline $\begin{array}{c}\text { ESPESSURAS } \\
\text { DE } \\
\text { ESMALTE }\end{array}$ & $\begin{array}{c}\text { EAIXDA3,5 } \\
(1 \mathrm{~mm})\end{array}$ & $\begin{array}{c}\text { EA1XDA3,5 } \\
(2 \mathrm{~mm})\end{array}$ & $\begin{array}{c}\text { EA2XDA3,5 } \\
(1 \mathrm{~mm})\end{array}$ & $\begin{array}{c}\text { EA2XDA } 3,5 \\
(2 \mathrm{~mm})\end{array}$ & $\begin{array}{c}\text { EA3XDA3,5 } \\
(1 \mathrm{~mm})\end{array}$ & $\begin{array}{l}\text { EA3XA3,5 } \\
(2 \mathrm{~mm})\end{array}$ & $\begin{array}{l}\text { EA3,5XA3,5 } \\
(1 \mathrm{~mm})\end{array}$ & $\begin{array}{l}\mathrm{EA} 3,5 \mathrm{XA} 3,5 \\
(2 \mathrm{~mm})\end{array}$ \\
\hline $0,2 \mathrm{~mm}(1)$ & $8,7( \pm 0,9)^{a}$ & $6,0( \pm 0,3)^{a}$ & $7,0( \pm 0,3)^{\mathrm{a}}$ & $3,2( \pm 0,8)^{\mathrm{A}}$ & $7,5( \pm 0,1)^{\mathrm{a}}$ & $1,6( \pm 0,3)^{A}$ & $8,1( \pm 0,5)^{a}$ & $3,1( \pm 0,6)^{A}$ \\
\hline $0,4 \mathrm{~mm}(2)$ & $6,8( \pm 0,4)^{\mathrm{a}}$ & $4,3( \pm 0,1)^{\mathrm{a}}$ & $3,5( \pm 0,2)^{\mathrm{a}}$ & $2,1( \pm 0,2)^{A}$ & $5,3( \pm 0,4)^{\mathrm{a}}$ & $1,4( \pm 0,2)^{A}$ & $7,4( \pm 0,3)^{a}$ & $4,4( \pm 0,1)^{a}$ \\
\hline $0,6 \mathrm{~mm} \mathrm{(3)}$ & $3,8( \pm 0,2)^{\mathrm{a}}$ & $2,7( \pm 0,3)^{\mathrm{A}}$ & $2,4( \pm 0,3)^{A}$ & $2,6( \pm 0,2)^{A}$ & $3,7( \pm 0,4)^{\mathrm{a}}$ & $2,5( \pm 0,2)^{\mathrm{A}}$ & $7,0( \pm 0,1)^{\mathrm{a}}$ & $5,6( \pm 0,1)^{\mathrm{a}}$ \\
\hline $0,8 \mathrm{~mm}(4)$ & $3,1( \pm 0,5)^{\mathrm{A}}$ & $2,3( \pm 0,1)^{A}$ & $2,2( \pm 0,1)^{\mathrm{A}}$ & $1,0( \pm 0,1)^{\mathrm{A}}$ & $3,2( \pm 0,2)^{\mathrm{A}}$ & $1,6( \pm 0,3)^{A}$ & $6,8( \pm 0,4)^{\mathrm{a}}$ & $5,5( \pm 0,3)^{\mathrm{a}}$ \\
\hline 1,0 mm (5) & $3,4( \pm 0,8)^{\mathrm{a}}$ & $2,4( \pm 0,2)^{\mathrm{A}}$ & $1,3( \pm 0,3)^{\mathrm{A}}$ & $2,3( \pm 0,2)^{\mathrm{A}}$ & $2,1( \pm 0,2)^{A}$ & $2,0( \pm 0,4)^{A}$ & $5,8( \pm 0,1)^{a}$ & $5,1( \pm 0,0)^{\mathrm{a}}$ \\
\hline
\end{tabular}

Fonte: UFPA, 2017.

Letra maiúscula (A) para valores compativeis com a escala VITA $(\Delta E \leq 3,3)$; Letra minúscula (a) para valores não compatíveis com a escala VITA $(\Delta E>3,3)$;

\section{DiscuSSÃo}

As resinas de esmalte e dentina foram desenvolvidas com o objetivo de aproximar as restaurações das características ópticas dos dentes naturais. Translucidez e opacidades distintas são os fatores determinantes para a sensação de profundidade, fator crucial para obtenção de uma restauração com aspecto semelhante à de um dente hígido ${ }^{14}$. Tem sido preconizada a utilização destas resinas de forma estratificada, substituindo no preparo cavitário as regiões de dentina e esmalte, de acordo com os diferentes níveis de translucidez que estas apresentam $^{15}$.

Em 2015, Shadman et a ${ }^{16}$ sugeriram que a espessura mínima de $0,8 \mathrm{~mm}$ em restaurações de porcelana (IPS e.max Press) era capaz de mascarar alterações de cores e promover compatibilidade de cor, segundo análise com espectrofotômetro. Porém, não foi encontrado um protocolo a ser seguido demonstrando uma espessura mínima no caso de restaurações com estratificação de resina composta. A localização e profundidade das cavidades são variáveis, por isso, se faz necessário diversificar também as espessuras de incrementos das resinas. Neste estudo, foram feitas diferentes espessuras de corpos de prova de resina para esmalte (de $0,2 \mathrm{~mm}$ a $1 \mathrm{~mm}$ ) e para dentina ( $1 \mathrm{~mm} \mathrm{e} 2 \mathrm{~mm}$ ), que foram avaliadas individualmente simulando situações clínicas diversas, com o objetivo de avaliar o desempenho da cor.

O aparelho utilizado para leitura dos corpos de prova foi um espectrofotômetro de refletância. Este dispositivo ainda não tem o seu uso popularizado, mas pode ser um excelente auxiliar na escolha da cor, pois, mede o montante de luz refletida por uma superfície como uma função de comprimento de onda, resultando em um espectro de refletância ${ }^{12}$. É um aparelho portátil, sem fio, opera com bateria e tem a capacidade de apresentar resultados mais precisos em relação à compatibilidade de cores ${ }^{13}$. Foi desenvolvido e comercializado para o uso clínico odontológico, com o objetivo de minimizar a subjetividade da estimativa visual de cor e para leitura de acordo com a percepção do olho humano. Os valores fornecidos pelo equipamento são $\Delta \mathrm{E} ; \mathrm{L} ; \mathrm{C} ; \mathrm{H} \mathrm{e}\left({ }^{*}\right)$ este último é uma escala de um a três asteriscos que avalia o 
nível de coincidência da cor analisada com a escala de cores Vita Classical, sendo um asterisco o menor nível de compatibilidade e três o máximo. Neste estudo, apenas os valores de $\Delta \mathrm{E}$ foram considerados para análise.

Estudos anteriores mostraram que diferenças nos componentes básicos das resinas como partículas de carga $^{17,18}$, matriz orgânica ${ }^{19}$, sistema fotoiniciador ${ }^{20}$ e a espessura da camada de material ${ }^{19,21}$ podem influenciar as propriedades ópticas, cor, e translucidez das resinas compostas, durante ou após a polimerização. No sistema Opallis, Guiraldo et al. ${ }^{22}$ afirmam que cores mais claras tendem a permitir maior passagem de luz pois, os pigmentos das cores mais escuras, como o laranja ou o vermelho, podem absorver mais luz que pigmentos claros. Embora Hyun et al. ${ }^{23}$ afirmem que camadas mais finas de resina composta sejam capazes de compensar a discrepância de cor de forma mais conservadora, sem a necessidade de um maior desgaste, para permitir a estratificação com resinas de dentina e esmalte. Este estudo mostrou que na interação de dentina DA2 com as cores de resina para esmalte EA1, EA2 e EA3, sob qualquer espessura (exceto na resina EA1 de 0,2 mm), foi capaz de apresentar similaridade com a escala de cores VITA, desde que a espessura utilizada de resina de dentina fosse de $2 \mathrm{~mm}$.

Apesar de Vichi et al. ${ }^{24}$ sugerirem que a dentina determina a cor no dente, notou-se neste estudo que a resina de esmalte pareceu ser mais determinante na influência e determinação de compatibilidade de cores, já que para a leitura com o espectrofotômetro o equipamento era calibrado de acordo com a cor da resina de esmalte, quando esta se encontrava sobreposta à resina de dentina. Vale destacar que os valores de EA3,5 foram bastante discrepantes em relação às outras saturações de esmalte. No presente estudo, não foi possível determinar o motivo de tal ocorrência. Pode-se apenas sugerir que nesta saturação $(A 3,5)$, a fabricação desta resina de esmalte não conseguiu estabelecer similaridade com a estabelecida pela escala VITA, provavelmente pelo percentual de corante ou da própria pigmentação amarela conferida pela canforoquinona, já que por se tratar da mesma resina supõe-se que o tipo e percentual em peso e volume de partículas seja o mesmo. Outras análises como a realizada por Fahl et al. ${ }^{25}$ demonstraram compatibilidade de cor da resina Opallis com a escala de cores VITA, porém apenas na cor A3. Sugere-se, portanto, a necessidade de estudos complementares de caracterização química, como alternativa para elucidar tal comportamento.

A prática clínica de uso de resinas de esmalte e dentina pode ser considerada recente, isto se reflete no desconhecimento do modo adequado de utilização por muitos profissionais e alunos de graduação. Este estudo evidenciou que a utilização de apenas resinas de dentina não seria viável já que revelou uma grande incompatibilidade com os padrões especificados. O mesmo insucesso seria obtido, se inadvertidamente o profissional utilizasse apenas as resinas de esmalte, em casos onde houvesse perda simultânea de esmalte e de dentina. Por outro lado, as associações destas duas resinas não obtiveram o sucesso esperado em todas as variações de espessura, principalmente, quando a espessura de dentina era de apenas $1 \mathrm{~mm}$.

A técnica restauradora com resina composta de forma estratificada ainda é desconhecida por muitos profissionais, e gera algumas dificuldades no seu aprendizado. A busca por um protocolo clínico que facilite ou possibilite a padronização de seu uso, justifica este tipo de estudo. Temos ainda que considerar que não há equivalência de cor entre todas as marcas comerciais e que nem todas disponibilizam materiais que substituam as propriedades óticas do esmalte e dentina.

Novos estudos devem ser realizados avaliando outras espessuras de ambos os materiais ou empregando camadas de dentina ou esmalte natural como referência.

\section{Conclusão}

De acordo com os dados analisados, pode-se sugerir que as resinas da marca Opallis apresentam necessidade de associação entre resinas de esmalte e dentina, para que atinjam níveis de excelência e conformidade de cor com o padrão estabelecido pela escala de cores Vita Classical, e que esta compatibilidade depende ainda da espessura da camada de resina. 


\section{REFERÊNCIAS}

1. Araújo EP, Filho LAR, Brum GT, Caldo TAS. Fechamento de diastemas com restaurações diretas de resina composta. Relato de caso clínico. Rev Gestão \& Saúde. 2009; 3(1):33-8.

2. Carmona D. Brincando com a luz. Artes médicas: São Paulo, 2006.

3. Ghersel ELA, Rodrigues CRMD, Gherse H. Entendendo as cores - parte 1. R Dental Press Estet. 2007 abr/mai/jun; 4(2):41-6.

4. Oliveira JOB, Cioffi MS, Cesnik RM, Florez FL, Bagnato VS, Corrêa SDR, et al. Comparative assessment of the organization of the colors of the Vita Classical color pallet by digital images and visual analysis for dental bleaching. Lasers in Dentistry. 2009 feb; 7162:20.

5. Moura BA, Silva CC. A teoria dos estados da luz: considerações sobre alguns papéis das hipóteses na óptica newtoniana. Associação de Filosofia e História da Ciência do Cone Sul. 2008; 5:91-100.

6. Sproull, RC. Color matching in dentistry. Part I. The three dimensional nature of color. J Prosthet Dent. 1973; 29(4):416-24.

7. Hirata R, Ampessan RL, Liu J. Reconstrução de dentes anteriores com resina composta: Uma sequência de escolha e aplicação de resinas. J Bras Clin Estet Odontol. 2001 jan./fev;5(25).

8. Higashi C, Hirata R, Masotti AS. Simplificando o uso de resinas compostas em dentes posteriores. Rev Dental Press de Estética. 2004; 1(1):18-34.

9. Silva JMF, Rocha DM, Kimpara ET, Uemura ES. Resinas compostas: estágio atual e perspectivas. Revista Odonto. 2008 dez; 16:101.

10. Júnior EJS, Brandt WC, Hernandez CP, Sinhoreti MAC. Fotoativação na atualidade: conceitos e técnicas clínicas. Int J Braz Dent, 2014 jun;10(2):188-97.

11. Junior PCM, Cardoso RM, Magalhães BG, Guimarães RP, Silva CHV, Beatrice LCS. Selecionando corretamente as resinas compostas. Int J Dent, 2011 jun; 10(2):91-6.

12. Amore R, Netto CA, Lewgoy HR, Siqueira EP, Carmo ARP. Cor em dentística - Desmistificando os principais conceitos. Int J Braz Dent, 2011 abr/jun; 10(2):91-6.

13. Kalantari MH, Ghoraishian SA, Mohaghegh M. Evaluation of accuracy of shade selectio using two spectrophotometer systems: Vita Easyshade and Degunent Shadepilot Europ J Dent. 2017;11(2):196-200.

14. Jardim PS, Miranda CB, Candido MSM, Lima DM. Análise comparativa da translucidez do esmalte e das diferentes resinas compostas microparticuladas. Cienc Odontol Bras. 2002 dez; 5(3):19-22.

15. Correia A, Oliveira MA, Silva MJ. Conceitos de estratificação nas resinas de dentes anteriores com resinas compostas. Rev Port Estomatol Med Dent Cir Maxilofac, 2005 set; 3:171-8.
16. Shadman N, Kandi SG, Ebrahimi SF, Shoul MA. "The minimum thickness of a multilayer porcelain restoration required for masking severe tooth discoloration". Dent Res J. 2015 nov-dec;12(6):562-8.

17. Suh Y, Ahn J, Ju S, Kim K. Influences of filler content and size on the color adjustment potential of nonlayered resin composites. Dent Mater J. 2017; 36(1):35-40.

18. Perez MM, Hita-iglesias C, Ghinea R, Yebra A, Pecho OE, ionescu AM, et. al. Optical properties of supra-nano spherical filled resin composites compared to nano filled, nano-hybrid and micro-hybrid composites. Dent Mater J. 2016; 35(3):353-59.

19. Hadis MA, Tomlins PH, Shortall AC, Palin WM. Dynamic monitoring of refractive index change through photoactive resins. Dent Mater. 2010 Nov; 26(11):1106-12.

20. Hadis MA, Shortall AC, Pali WM. Competitive light absorbers in photoactive dental resin-based materials. Dent Mater. 2012 Aug;28(8):831-41.

21. Melo TS, Kano P, Araújo EM. Avaliação e reprodução cromática em odontologia restauradora: Parte I. 0 mundo das cores. Int. J. Braz. Dent. 2005 abr./jun;1(2):95-104.

22. Guiraldo RD, Consani S, Consani RL, Berger SB, Mendes WB, Sinhoreti MA. Light energy transmission through composite influenced by material shades. Bull Tokyo Dent Coll. 2009; 50(4):183-90.

23. Hyun H, Christoferson CK, Pfeifer CS, Felix C, Ferracane JL. Effect of shade, opacity and layer thickness on light transmission through a nano-hybrid dental composite during curing. J Esthet Restor Dent. 2017; 29:362-7.

24. Vichi A, Fraioli A, Davidson CL, Ferrari M. Influence of thickness on color in multi-layering technique. Dent Mater. 2007;23(12):1584-9.

25. Fahl NJ. A solution for everyday direct restorative challenges. J Cosme Dent. 2010; 26(3):56-67. 